\title{
Symmetries in Evolving Space-Time and Their Connection to High-Frequency Gravitational Wave Production
}

\author{
Andrew Walcott Beckwith \\ Physics Department, College of Physics, Chongqing University, Chongqing, China \\ Email: Rwill9955b@gmail.com, abeckwith@uh.edu
}

How to cite this paper: Beckwith, A.W. (2018) Symmetries in Evolving Space-Time and Their Connection to High-Frequency Gravitational Wave Production. Journal of High Energy Physics, Gravitation and Cosmology, 4, 492-503.

https://doi.org/10.4236/jhepgc.2018.43027

Received: May 7, 2018

Accepted: July 3, 2018

Published: July 6, 2018

Copyright $\odot 2018$ by author and Scientific Research Publishing Inc. This work is licensed under the Creative Commons Attribution International License (CC BY 4.0).

http://creativecommons.org/licenses/by/4.0/

\begin{abstract}
We claim that the linking of a shrinking prior universe to our own via a wormhole bridge solution of about ten to the minus forty four power seconds permits the formation of a short-term quintessence scalar field. Symmetries allow for creating high-frequency gravitational waves at the onset of inflation, which has consequences in our present cosmological era. This instantaneous energy transfer between prior to present universes permits relic graviton production which we claim is a viable candidate for future propulsion technologies in space craft design. The Big Bang started as the passage of thermal energy from an existing universe into ours resulting in another Big Bang, and helps us understand how a graviton burst can occur in the first place.
\end{abstract}

\section{Keywords}

Wormhole, High-Frequency Gravitational Waves (HGW), Symmetry, Causal Discontinuity

\section{Introduction}

We begin first with a restatement of the physics leading to a wormhole solution for transfer of vacuum energy from a prior universe to today's expanding universe. We then argue that such a vacuum-energy transfer is a necessary condition for forming short-term quintessence scalar fields. This allows us to form quintessence scalar-field behavior that is consistent with the $w=-1$ value for the ratio of pressure and density, which is well within the known red shift $z=1100$ cosmic microwave background barrier. We should note that initially, $w=0$ at the initial onset of the big bang, also that the CMB radiation cutoff region is 380 to 400 thousand years after the big bang [1] [2]. At that time, we are 
able to start seeing a separation of photons and matter, so we can observe stellar astrophysical processes reliably. After restating how a wormhole solution forms, results heavily depend on the scalar field turning into a real quality, after the initial phases of inflation, as well as rapidly damping out as the vacuum energy creates emergent field conditions, allowing for the relic production of gravitons. The existence of the solution of the Wheeler-DeWitt equation with a pseudo-time-like component provides an additional symmetry to space-time evolution, which is broken by the chaotic regime of the scale factor.

This leads to a bifurcation in the evolution of the quintessence scalar field that comes from initially zero value for the scalar field in the initial onset of inflation. In the present day era, the cosmological data set leads us to conclude that the rate of expansion of the universe is actually increasing. In between these two zero values of the scalar field, we have non-zero values of the scalar field. This, with the Seth Lloyd model [3] of the universe as a quantum computing device, permits us to specify a shift to high-frequency gravitational waves. This is a way to keep a finite but large number of computational bits of information for modeling universe expansion, regardless of how large the universe becomes in the present to far future.

\section{How a Wormhole Forms}

Lorentzian wormholes [1] [2] have been modeled quite thoroughly. Visser (1995) [4] states that the wormhole solution does not have an event horizon hiding a singularity, i.e., there is no singularity in the wormhole that is held open by dark energy. We are presenting a wormhole purely as a bridge between a prior to a present universe, as Crowell (2005) [5] refers to in his reference on quantum fluctuations of spacetime. The equation for thermal/vacuum energy flux, which leads to a wormhole, uses a pseudo-time-like space coordinate in a modified Wheeler-De Witt equation for a bridge between two universes. We also state that the wormhole solution is dominated by a huge vacuum-energy value.

To show this, we use results from Crowell (2005) [5] on quantum fluctuations in space-time. This gives a model from a pseudo-time-component version of the Wheeler-De Witt equation, [5] [6] with use of the Reinssner-Nordstrom metric [1] [2] to help us obtain a solution that passes through a thin shell separating two space-times. The radius of the shell, $r_{0}(t)$ separating the two spacetimes is of length $l_{P}$ in approximate magnitude, leading to a domination of the time component for the Reissner-Nordstrom metric [1] [2] [5]

$$
\mathrm{d} S^{2}=-F(r) \cdot \mathrm{d} t^{2}+\frac{\mathrm{d} r^{2}}{F(r)}+\mathrm{d} \Omega^{2}
$$

This has: [5]

$$
F(r)=1-\frac{2 M}{r}+\frac{Q^{2}}{r^{2}}-\frac{\Lambda}{3} \cdot r^{2} \underset{T \rightarrow 10^{32} \mathrm{Kelvin} \sim \infty}{\longrightarrow}-\frac{\Lambda}{3} \cdot\left(r=l_{P}\right)^{2}
$$

This assumes that the cosmological vacuum energy parameter has a temperature-dependence as outlined by Park (2003) [7], leading to 


$$
\frac{\partial F}{\partial r} \sim-2 \cdot \frac{\Lambda}{3} \cdot\left(r \approx l_{P}\right) \equiv \eta(T) \cdot\left(r \approx l_{P}\right)
$$

a wave-functional solution to a Wheeler-De Witt equation bridging two space-times. This solution bridging two space-times is similar to that being made between these two space-times with "instantaneous" transfer of thermal heat, as given by Crowell (2005) [5]

$$
\Psi(T) \propto-A \cdot\left\{\eta^{2} \cdot C_{1}\right\}+A \cdot \eta \cdot \omega^{2} \cdot C_{2}
$$

This has $C_{1}=C_{1}(\omega, t, r)$ as a cyclic and evolving function in terms of frequency, time, and spatial function, with the same thing describable about $C_{2}=C_{2}(\omega, t, r)$, with $C_{1}=C_{1}(\omega, t, r) \neq C_{2}(\omega, t, r)$. The upshot of this is that a thermal bridge exists between a shrinking prior universe collapsing to a singularity and an expanding universe expanding from a singularity, with an almost instantaneous transfer of heat from that prior universe to today's cosmology. The thermal bridge being modeled as a wormhole is a necessary and sufficient condition for an almost instantaneous transferal mechanism of matter-energy to today's universe from a prior cosmological collapse. We get this by assuming that the absolute value of the five-dimensional "vacuum state" parameter varies with temperature $T$, as Beckwith (2007) [8] writes

$$
\left|\Lambda_{5-\mathrm{dim}}\right| \approx c_{1} \cdot\left(1 / T^{\alpha}\right)
$$

in contrast with the more traditional four-dimensional version, minus the minus sign of the brane-world theory version. The five-dimensional version is based on brane theory and higher dimensions, whereas the four-dimensional version is linked to more traditional de Sitter space = time geometry, as given by Park (2003) [7]

$$
\Lambda_{4-\operatorname{dim}} \approx c_{2} \cdot T^{\beta}
$$

If we look at the range of allowed upper bounds for the cosmological constant, there exists a difference in values between what Park (2003) [7] predicts-an almost infinite value - to a much lower value given by Barvinsky (2006) [9], which is 360 times the square of Planck's mass. The difference in these two values is commensurate with the existence of a symmetry-breaking phase transition, where we predict spin two gravitons are released, and also when we observe axion domain wall decay. We should note that this assumes that a release of gravitons occurs, which leads to a removal of graviton-energy-stored contributions to this cosmological parameter, with $m_{\mathrm{P}}$ as the Planck mass, i.e., the mass of a black hole of "radius" on the order of magnitude of Planck length $l_{\mathrm{P}} \sim 10^{-35} \mathrm{~m}$.

This leads to Planck's mass $m_{P} \approx 2.17645 \times 10^{-8}$ kilograms, as alluded to by Barvinsky (2006) [9]

$$
\Lambda_{4-\operatorname{dim}} \propto c_{2} \cdot T \underset{\text { graviton-production }}{\longrightarrow} 360 \cdot m_{P}^{2} \ll c_{2} \cdot\left[T \approx 10^{32} \mathrm{~K}\right] .
$$

Right after the gravitons are released one still sees a drop-off of temperature contributions to the cosmological constant. Then we can write, for small time values, $t \approx \delta^{1} \cdot t_{P}, \quad 0<\delta^{1} \leq 1$, and for temperatures sharply lower than 
$T \approx 10^{12}$ Kelvin, as commented on by Beckwith (2007), [8] that there exists a positive integer $n$, which leads to a sharp phase-transition drop for temperature values as we approach Planck's time interval, $t_{P} \sim 10^{-44}$ seconds.

After the exit of vacuum "thermal" energy from the mouth of the wormhole bridge between a prior universe and our universe, within a Planck time interval, we observe a decrease in values of the cosmological constant in the four-dimensional world and an increase in the absolute value of the "vacuum energy" in the five-dimensional brane world. However, their absolute magnitudes are approximately the same after the Planck time interval.

At and before this region of relative equivalence of these magnitudes (the cosmological constant in four space and the absolute value of vacuum energy in five dimensional brane worlds), a scale-factor discontinuity region (referred to later in this paper) exists. In the region of time and space before we traverse this thermal/spatial scale factor discontinuity region, we have $10^{10}$ or so bits of "information".

After traversing this thermal/spatial scale-factor discontinuity region, it rapidly increase to $10^{120}$ or so bits of information, due to an increase in complexity of the cosmological space-time structure. The dividing line between these two regions of complexity is shown by the behavior of the four-dimensional cosmological constant energy and the five dimensional "vacuum energy" expressions discussed above.

What we do state, however, is that in the region before this discontinuity region, we have less than or equal to $10^{10}$ bits of "information". This changes to a four-dimensional "cosmological constant" energy far smaller than the magnitude of the five-dimensional brane word vacuum energy, becoming $10^{120}$ bits of actual cosmological information and leading to the large-scale creation of structure in our universe.

$$
\frac{\Lambda_{4-\operatorname{dim}}}{\left|\Lambda_{5-\operatorname{dim}}\right|}-1 \approx \frac{1}{n}
$$

The transition outlined in Equation (7) above has a starting point with extremely high temperatures created by a vacuum-energy transfer between a prior universe and our present universe, as outlined by Equation (3) and Equation (4) above. Whereas the regime where we look at an upper bound to vacuum energy in four dimensions is outlined in Equation (8) above. This wormhole solution is a necessary and sufficient condition for thermal transfer of heat from that prior universe to allow for graviton production under relic inflationary conditions.

Claim 1: The Following Are Equivalent

1) There exists a Reisnner-Nordstrom Metric with $-F(r) \mathrm{d} t^{2}$ dominated by a cosmological vacuum energy term, $(-\Lambda / 3)$ times $\mathrm{d} t^{2}$, for early-universe conditions in the time-range less than or equal to Planck's time $t_{P}$.

2) A solution for a pseudo-time-dependent version of the Wheeler-De Witt equation exists with a wave function $\Psi(r, t, T)$, forming a wormhole bridge between two universe domains, with $\Psi(r, t, T)=\Psi(r,-t, T)$ for a region of 
space-time before signal causality discontinuity, and for times $|t|<t_{P}$.

3) The heat flux-dominated vacuum energy value given by $\Psi(r, t, T)$ contributes to a relic graviton burst, in a region of time less than or equal to Planck's time $t_{P}$.

The proof of claim 1 is referenced via an article, Beckwith (2007). [8] This claim establishes the structure we outline as to our causal discontinuity approach to wormholes [1] [2] [5].

\section{Presenting Evidence for Causal Discontinuity Due to the Transfer of Thermal-Based Vacuum Energy Implied By the Wheeler-De Witt Equation Wormhole Solution}

Begin first by presenting a version of the Friedmann equation given by Frampton (2007) [10]:

$$
(\dot{a} / a)^{2}=\frac{8 \pi G}{3} \cdot\left[\rho_{\text {rel }}+\rho_{\text {matter }}\right]+\frac{\Lambda}{3}
$$

We argue that the existence of such a nonlinear equation for early-universe scale-factor evolution introduces a de facto "information" barrier between a prior universe, which, as we argue, can only include thermal bounce input to the new nucleation phase of our present universe. To see this, we can turn to Dr. Dowker's paper on causal sets [11]. These require the following ordering with a relation $\prec$, where we assume that initial relic space time is replaced by an assembly of discrete elements, so as to create, initially, a partially ordered set [12] [13], $C$ :

1) If $x \prec y$ and $y \prec z$, then $x \prec z$.

2) If $x \prec y$ and $y \prec x$, then $x=y$ for $x, y \in C$.

3) For any pair of fixed elements $x$ and $z$ of elements in $C$, the set $\{y \mid x \prec y \prec z\}$ of elements lying in between $x$ and $z$ is finite.

Items (1) and (2) give us that we have $C$ as a partially ordered set [12] [13] and the third item permits local finiteness. When combined with as a model for how the universe evolves via a scale factor equation, this permits us to write, after we substitute $a\left(t^{*}\right)<l_{P}$ for $t^{*}<t_{P}=$ Planck time, and $a_{0} \equiv l_{P}$, and $a_{0} / a\left(t^{*}\right) \equiv 10^{\alpha}$ for $\alpha \gg 0$ into a discrete equation model of Equation (5) leads to:

Claim 2: Using the Friedmann Equation For the Evolution of a Scale Factor $a(t)$, We Have a Non-Partially Ordered-Set Evolution of the Scale Factor with Evolving Time, Implying a Causal Discontinuity

We establish the validity of this formalism by rewriting the Friedman equation as follows:

$$
\left[\frac{a\left(t^{*}+\delta t\right)}{a\left(t^{*}\right)}\right]-1<\frac{\left(\delta t \cdot l_{P}\right)}{\sqrt{\Lambda / 3}} \cdot\left[1+\frac{8 \pi}{\Lambda} \cdot\left[\left(\rho_{r e l}\right)_{0} \cdot 10^{4 \alpha}+\left(\rho_{m}\right)_{0} \cdot 10^{3 \alpha}\right]\right]^{1 / 2} \underset{\Lambda \rightarrow \infty}{\longrightarrow} 0(10)
$$

So in the initial phases of the big bang, with a very large vacuum energy, we obtain the following relation, which violates (signal) causality. This is for any 
given fluctuation of time in the "positive" direction:

$$
\left[\frac{a\left(t^{*}+\delta t\right)}{a\left(t^{*}\right)}\right]<1
$$

We argue that the existence of such a violation of causality, as shown in the evolution of the scale factor (as given in the ratio of a scale factor at a later time divided by the same factor at a prior time). The fact that the scale factor is less than one argues for a break in information propagation from a prior universe to our present universe.

One relevant area of inquiry to be investigated in the future is the following: Is this argument valid if there is some third choice of set structure? (For instance, do self-referential sets fall into one category or another?) The answer to this, we think, lies in (entangled?) vortex structure of space-time similar to that generated in the laboratory by Ruutu (1996) [14]. Self-referential sets [15] may be part of the generated vortex structure and we will endeavor to find if this can be experimentally investigated. If the causal-set argument, and its violation via this procedure, holds, we have the view that what we are seeing is a space-time "drum" effect, with the causal discontinuity forming the head of a "drum" for a region of about $10^{10}$ bits of "information" before our present universe up to the instant of the big bang itself for a time region less than $t \sim 10^{-44}$ seconds in duration, with a region of increasing bits of "information" going up to $10^{120}$ due to vortex filament condensed matter style forming through a symmetry breaking phase transition.

\section{Seth Lloyd's Universe as a Quantum Computer Model with Modifications}

We use the formula given by Seth Lloyd (2002) [3] that defines the number of operations the "Universe" can "compute" during its evolution. Lloyd (2002) [3] uses the idea attributed to Landauer that the universe is a physical system with information processed over its evolutionary history. Lloyd also cites a prior paper where he attributes an upper bound to the permitted speed a physical system can have in performing operations in lieu of the Margolis/ Levitin theorem. He specifies a quantum mechanically given upper limit value (assuming $\mathrm{E}$ is the average energy of the system above a ground state value), obtaining a first limit of a quantum mechanical average energy bound value of

$$
[\text { \# operations } / \mathrm{sec}] \leq 2 E / \pi \hbar
$$

The second limit to this number of operations is strictly linked to entropy, due to considerations of limits to memory space, which Lloyd writes as

$$
\text { [\# operations }] \leq S(\text { entropy }) /\left(k_{B} \cdot \ln 2\right)
$$

The third limit, based on strict considerations of a matter-dominated universe, relates the number of allowed computations (operations) within a volume for the alleged space of a universe (horizon). Lloyd identifies this space-time vo- 
lume as $c^{3} \cdot t^{3}$, with $c$ the speed of light, and $t$ an alleged time (age) for the universe. We further identify $E$ (energy) $\sim \rho \cdot c^{2}$, with $\rho$ as the density of matter, and $\rho \cdot c^{2}$ as the energy density (unit volume). This leads to

$$
[\text { \# operations } / \mathrm{sec}] \leq \rho \cdot c^{2} \times c^{3} \cdot t^{3}
$$

We then can write this, if $\rho \sim 10^{-27} \mathrm{kil} / \mathrm{meter}^{3}$ and time as approximately $t \sim 10^{10}$ years. This leads to a present upper bound of

$$
\text { [\# operations] } \approx \rho \cdot c^{5} \cdot t^{4} \leq 10^{120}
$$

Lloyd further refines this to read [3]

$$
\text { \# operations }=\frac{4 E}{\hbar} \cdot\left(t_{1}-\sqrt{t_{1} t_{0}}\right) \approx\left(t_{\text {Final }} / t_{P}\right) \leq 10^{120}
$$

We assume that $t_{1}=$ final time of physical evolution, whereas $t_{0}=t_{P} \sim 10^{-43}$ seconds and that we can set an energy input by assuming, in early universe conditions, that $N^{+} \neq \varepsilon^{+} \ll 1$ and $0<N^{+}<1$. So that we are looking at a graviton-burst-supplied energy value of

$$
\begin{aligned}
E= & \left(V_{4-\text { Dim }}\right) \cdot\left[\rho_{\text {Vac }}=\frac{\Lambda}{8 \pi G}\right] \\
& \sim N^{+} \cdot\left[\rho_{\text {graviton }} \cdot V_{4-\text { vol }} \approx \hbar \cdot \omega_{\text {graviton }}\right]
\end{aligned}
$$

Furthermore, assuming the initial temperature is within the range of $T \approx 10^{32}-10^{29}$ Kelvin, we have a Hubble parameter defined along the route specified by Lloyd [3]. This is in lieu of time $t=1 / H$, a horizon distance defined as $\approx c / H$, and a total energy value within the horizon as Energy (within the horizon)

$$
\approx \rho_{C} \cdot c^{3} /\left(H^{4} \cdot \hbar\right) \approx 1 /\left(t_{P}^{2} \cdot H\right)
$$

And this for a horizon parameter Lloyd (2002) defines as [3]

$$
H=\sqrt{8 \pi G \cdot\left[\rho_{\text {crit }}\right] / 3 \cdot c^{2}}
$$

And a early universe

$$
\rho_{\text {crit }} \sim \rho_{\text {graviton }} \sim \hbar \cdot \omega_{\text {graviton }} / V_{4-\mathrm{Vol}}
$$

Then

$$
\begin{aligned}
\text { \# operations } & \approx 1 /\left[t_{P}^{2} \cdot H\right] \approx \sqrt{V_{4-\mathrm{Vol}}} \cdot t_{P}^{-2} / \sqrt{\left[8 \pi G \hbar \omega_{\text {graviton }} / 3 c^{2}\right]} \\
& \approx[3 \ln 2 / 4]^{4 / 3} \cdot\left[S_{\text {Entrophy }} / k_{B} \ln 2\right]^{4 / 3}
\end{aligned}
$$

Claim 3: The Number of Allowed Operations In the Evolution of the Universe Specifies a Relationship between an Evaluated Volume for Space-Time and Upper Limits of Released Relic-Graviton Frequencies

This is proved by appealing to Equation (21) above. Next, we will examine the existence of certain symmetries in the scalar field itself [1] [2] [16].

\section{Formation of the Scalar Field, Bifurcation Results}

Start with Padamans's formulas (2006) [16]: 


$$
V(t) \equiv V(\varphi) \sim \frac{3 H^{2}}{8 \pi G} \cdot\left(1+\frac{\dot{H}}{3 H^{2}}\right)
$$

and

$$
\varphi(t) \sim \int \mathrm{d} t \cdot \sqrt{\frac{-\dot{H}}{4 \pi G}}
$$

If $H=\dot{a} / a$, Equation (23) gives us zero scalar field values at the beginning of quantum nucleation of a universe. At the point of accelerated expansion (due to the final value of the cosmological constant), it also gives an accelerating value of the cosmological scale-factor expansion rate. We justify this statement by using early-universe expansion models, which have $a\left(t_{\text {INITIAL }}\right) \sim \mathrm{e}^{H \cdot t}$. This leads to the derivative of $H=\dot{a} / a$ going to zero. This is similar to present-time development of the scalar factor along the lines of $a\left(t_{\text {later }}\right) \sim \mathrm{e}^{(\Lambda[\text { present-day }] \cdot t)}$, also leading to the derivative of $H=\dot{a} / a$ going to zero. When both situations occur, we have the scale factor $\phi=0$. Between initial and later times, the scale factor no longer has exponential time dependence, due to it growing far more slowly, leading to $\phi \neq 0$.

Both regimes as specified by Equation (23) above lead to zero values for a quintessence scalar field. But it does not stop there. We will show later that in actuality, the scalar field likely damps out far before the CMBR barrier value of expansion when $Z=1100$, about 380,000 to 400,000 years after the big bang [1] [2].

Claim 4: We Observe That the Scalar Field $\phi(t)$ Is Zero at the Onset of the Big Bang, and Also Is Zero During the Present Cosmological Era

This scalar "quintessence" field [1] [2] [5] [16] [17] is non zero in a brief period of time right after the inflationary era.

We show this by noting that in Equation (22), the time derivative of $H=\dot{a} / a$ goes to zero when both the scale factors $a(t($ initial $)) \sim \exp (H \cdot t($ initial $))$, and $a(t($ later $)) \sim \exp (\Lambda($ today $) \cdot t($ later $))$. The exponential scale factors in both cases (the initial inflationary environment and the present era) lead to the time derivative of the $H=\dot{a} / a$ expression in Equation (23) going to zero.

Sub point to claim 4: The existence of two zero values of the scalar field $\phi(t)$ at both the onset and at a later time implies a bifurcation behavior for modeling quintessence scalar fields. This is due to the non-zero $\phi(t)$ values right after the initiation of inflation.

How do we construct high-frequency gravitational waves from all of this? Note that in Equation (21) above, that we have the existence of a denominator of the right hand side of the equation with the square of the Planck's time. It so happens that inflation is characterized with a rapid buildup of space time volume. Dr. Smoot (2007) [18] specified initial computational bits of information transferred on the order of $10^{8}$ to $10^{10}$ bits of computation, expanding up to $10^{120}$ shortly after the initiation of the big bang itself. This creation of additional degrees of freedom is in tandem with breaching the scale factor discontinuity men- 
tioned in Claim 2 above. When we get to this regime of scale factor discontinuity, we get into the physics discussed by the claim given below.

Claim 5: Unless the frequency $\omega_{\text {graviton }}$ in Equation (21) becomes large $\left(\sim 10^{3}\right.$ $\mathrm{Hz}$ or higher), the number of operations could effectively go to $10^{1000}$ or higher.

How do we show this? One would need to have very large gravitational frequency range, with high frequency gravitational waves, in order to break the effects of a tiny Planck time interval $t_{P}^{-2} \sim 10^{86} \mathrm{sec}^{-2}$ put in the number of operations. So that instead of Equation (21) bounded by $10^{120}$, as the volume increased, the number of degrees of freedom of operations could become almost infinite.

This last claim - combined with the discussion right after Equation (11) above re the initial "drum head" model for a bounded region of space bracketed by causal discontinuity regions-constitutes our working model of an information-based model of cosmology that we expect will yield falsifiable experimental criteria.

\section{Smoot's Information Theory/Cosmology Conclusions at Paris Colloquium (2007 “D. Chalonge" School)}

At the "D. Chalonge" school presentation done by Dr. Smoot (2007) [18], he stated the following information-theory processing bits levels, which are due to different outlined physical processes. The following is Dr. Smoot's preliminary analysis of information content in the observable universe: [18]

1) Physically observable bits of information possibly generated in the Universe: $10^{180}$

2) Holographic principle-allowed bits (states) in the evolution (development) of the Universe: $10^{120}$

3) Initially available bits (states) given to us to work with at the onset of the inflationary era: $10^{10}$

4) Observable bits of information present due to quantum/statistical fluctuations: $10^{8}$

Our guess is as follows: the thermal flux implied by the existence of a wormhole accounts for perhaps $10^{10}$ bits of information. These could be transferred via a wormhole solution from a prior universe to our present, per Equation (4) above. So there could be perhaps $10^{120}$ minus $10^{10}$ bits of information temporarily suppressed during the initial bozonification phase of matter right at the onset of the big bang itself. Then the degrees of freedom of our initial cosmological environment dramatically dropped during the beginning of the descent of temperature from about $T \approx 10^{32}$ Kelvin to at least three orders of magnitude. This drop in temperature occurs as we move out from an initial red shift of $z \approx 10^{25}$ to $z \approx 10^{25}$ to a far smaller value of the red shift. A good guess as to what is going on is embodied in figure 28.1 of the book by Volovik (2003) [19], offering a condensed matter analogy to current cosmology, where the formation of topological cosmic defects according to the Kibble-Zurek hypothesis may be in tandem with the growth of cosmological bits of information from a low number of 
$10^{10}$ to $10^{120}$ today. This is similar to the growth in baryons of up to $10^{80}$ in the modern era. The formation of complexity of structure as given in figure 28.1, cited above, occurs at the same time as the bit complexity reaches its present value of nearly $10^{120}$.

\section{Conclusions}

So far, what we have established is a working model for an information theory based model of cosmological evolution with a lot of symmetry arguments thrown in. The approach is novel, leading to a new way of looking at CMBR space/volume and what it is, relative to bits of "information" computed during the course of cosmological evolution. This also permits a template for realistic models of a graviton burst.

As for future research, we should delineate in more detail what would be transferred-possibly by entanglement information transfer from a prior universe to our own. We should also understand how additional bits of information came to be in the present Universe. All of this would tie in with an accurate physical understanding of the points raised in Section 5 above. We would like to see-partly using the rich lore on liquid helium as outlined by Kopik (1993) [20] - if there is a way to experimentally determine if the growth and the relative increase in structure and bits of "information" are in some sense connected with a cosmological equivalent to the vortex reconnection process outlined in liquid helium experiments. In particular, the author is convinced that the fifth claim as given above is fundamental physics, and that as we have a growing volume during inflation, this needs to be investigated-hopefully in ways that lead to falsifiable computational quantum computer models of the universe, and to their connections to initial inflation physics.

We should mention that the High frequency gravitational wave regime is potentially accessible. A good write up as to this is given in [21] below.

In addition, we have that LIGO has made several gravitational wave discoveries. [22] is the first one, as is [23] and [24]. These establish basic working assumptions needed as far as gravitational wave astronomy data sets, whereas [21] is for the different regime of gravitational wave astronomy needed as far as higher frequencies, and different strength of GW than what is done by LIGO.

Finally, [25] establishes if we can discriminate between different models of gravity, whether these are scalar-tensor, general relativity, or some variant of quantum gravity, which may be identifiable if we have data sets to make proper evaluation.

In all this is a first step; in terms of identification of basic foundations of gravitational wave astronomy we hope is pursued in the new future.

We also wish to mention that additional issues brought up in [26] and [27] should be adhered to, in terms of experimental inquiry.

\section{Acknowledgements}

The author would like to acknowledge the contributions of Amara D. Angelica 
for stimulating discussions of the concepts and copyediting.

This work is supported in part by National Nature Science Foundation of China grant No. 11375279.

\section{References}

[1] Ryden, B. (2017) Introduction to Cosmology. 2nd Edition, Cambridge University Press, Cambridge.

[2] Jerzy Plebasnki, J. and Krasinki, A. (2007) An Introduction to General Relativity and Cosmology. Cambridge University Press, Cambridge.

[3] Lloyd, S. (2002) Computational Capacity of the Universe. Physical Review Letters 88, Article ID: 237901. https://doi.org/10.1103/PhysRevLett.88.237901

[4] Visser, M. (1995) Lorentzian Wormholes, from Einstein to Hawking. AIP Press, Woodbury, New York.

[5] Crowell, L. (2005) Quantum Fluctuations of Space Time. World Scientific Series in Contemporary Chemical Physics, Volume 25. World Scientific Publishing Company, Singapore.

[6] Hamber, H. and Williams, R. (2011) Discrete Wheeler-DeWitt Equation. Physical Review D, 84, Article ID: 104033. https://doi.org/10.1103/PhysRevD.84.104033

[7] Park, D.K., Kim, H. and Tamarayan, S. (2002) Nonvanishing Cosmological Constant of Flat Universe in Brane World Senarios. Physics Letters B, 535, 5-10. https://doi.org/10.1016/S0370-2693(02)01729-X

[8] Beckwith, A. (2007) Symmetries in Evolving Space Time from Present to Prior Universes. arXiv:math-ph/0501028

[9] Barvinsky, A., Kamenschick, A. and Yu, A. (2006) Thermodynamics from Nothing: Limiting the Cosmological Constant Landscape. Physical Review D, 74, Article ID: 121502. https://doi.org/10.1103/PhysRevD.74.121502

[10] Frampton, P. and Baum, L. (2007) Turnaround in Cyclic Cosmology. Physical Review Letters, 98, Article ID: 071301.

[11] Dowker, F. (2005) Causal Sets and the Deep Structure of Space-Time. In: Akshtekar, A., Ed., 100 Years of Relativity, Space-Time Structure: Einstein and Beyond, World Press Scientific, Singapore, 445-464. https://doi.org/10.1142/9789812700988_0016

[12] Simovici, D. and Djeraba, C. (2008) Partially Ordered Sets. Mathematical Tools for Data Mining: Set Theory, Partial Orders, Combinatorics. Springer, Berlin.

[13] Schröder, B.S.W. (2003) Ordered Sets: An Introduction. Birkhäuser, Boston. https://doi.org/10.1007/978-1-4612-0053-6

[14] Ruutu, V., Eltsov, V., Gill, A., Kibble, T., Krusius, M., Makhlin, Y.G., Placais, B., Volvik, G. and Wen, Z. (1996) Vortex Formation in Neutron-Irradiated ${ }^{3} \mathrm{He}$ as an Analog of Cosmological Defect Formation. Nature, 382, 334-336. https://doi.org/10.1038/382334a0

[15] Soto-Andrade, J., Jaramillo, S., Gutierrez, C. and Letelier, J.-C. (2011) Ouroboros Avatars: A Mathematical Exploration of Self-reference and Metabolic Closure. MIT Press, Paris.

[16] Padmanabhan, T. (2006) An Invitation to Astro Physics. World Scientific Publishing Co. Pte. Ltd., Singapore. https://doi.org/10.1142/6010

[17] Tsujikawa, S. (2013) Quintessence: A Review. Classical and Quantum Gravity, 30, Article ID: 214003. https://doi.org/10.1088/0264-9381/30/21/214003 
[18] Smoot, G. (2007) Nobel Lecture: Cosmic Microwave Background Radiation Anisotropies: Their Discovery and Utilization. Reviews of Modern Physics, 79, 1-31. https://cdn.journals.aps.org/files/RevModPhys.79.1349.pdf

[19] Volovik, G. (2003) The Universe in a Helium Droplet. International Series of Monographs on Physics 117, Clarendon Press, Oxford.

[20] Koplik, J. and Levine, H. (1993) Vortex Reconnection in Superfluid Helium. Physical Review Letters, 71, 1375-1378. https://doi.org/10.1103/PhysRevLett.71.1375

[21] Li, F., Baker Jr., R.M.L., Fang, Z., Stephenson, G.V. and Chen, Z. (2008) Perturbative Photon Fluxes Generated by High-Frequency Gravitational Waves and Their Physical Effects. The European Physical Journal C, 56, 407-423. https://doi.org/10.1140/epjc/s10052-008-0656-9

[22] Abbott, B.P., et al. (2016) Observation of Gravitational Waves from a Binary Black Hole Merger. Physical Review Letters, 116, Article ID: 061102.

[23] Abbott, B.P., et al. (2016) GW151226: Observation of Gravitational Waves from a 22-Solar-Mass Binary Black Hole Coalescence. Physical Review Letters, 116, Article ID: 241103.

[24] Abbott, B.P., et al. (2017) GW170104: Observation of a 50-Solar-Mass Binary Black Hole Coalescence at Redshift 0.2. Physical Review Letters, 118, Article ID: 221101.

[25] Corda, C. (2009) Interferometric Detection of Gravitational Waves: The Definitive Test for General Relativity. International Journal of Modern Physics D, 18, 2275-2282. https://arxiv.org/abs/0905.2502 https://doi.org/10.1142/S0218271809015904

[26] Barcelo, C. and Visser, M. (1999) Transversable Wormholes from Massless Conformally Coupled Scalar Fields. Physics Letters B, 66, 127-134.

[27] Visser, M. (2002) Sakarov's Induced Gravity: A Modern Perspective. 